\title{
Re: "Talonavicular joint arthrodesis for the treatment of pes planus valgus in older children and adolescents with cerebral palsy"
}

\author{
Mohan V. Belthur
}

Received: 14 August 2009/Accepted: 28 September 2009/Published online: 16 October 2009

(C) EPOS 2009

Dear Editor,

I read with interest the article titled Talonavicular joint arthrodesis for the treatment of pes planus valgus in older children and adolescents with cerebral palsy, by C. A. Turriago et al., published in the June 2009 issue of the Journal of Children's Orthopaedics (pp. 179-183). I think they have made an important contribution to the existing literature regarding the management of severe planovalgus foot in children with cerebral palsy. However, I would like to request some clarifications from the authors:

1. In the abstract and the introduction section, they comment that the purpose of the article is to report on their experience with talonavicular joint arthrodesis for the treatment of severe valgus foot deformities. However, they have failed to define what "severe" means in their article. I would appreciate it if they could clarify their definition of a severe planovalgus foot.

2. In the clinical evaluation part of the results section they do not comment about whether their patients had brace intolerance. If so, did that improve after surgical correction of the planovalgus foot deformity? In our experience, most patients will have brace intolerance, and this improves after surgical correction of the planovalgus foot deformity.

3. They report that ankle valgus was diagnosed postoperatively in six patients. It was not clear whether it was detected by clinical examination or on AP radiographs of the ankle.

M. V. Belthur $(\bowtie)$

Shriners Hospitals for Children, 2825, Bellefontaine Street,

Houston, TX 77025, USA

e-mail: mvbelthur@yahoo.com
4. In relation to the radiographic evaluation section: other studies in relation to planovalgus foot deformity in children with cerebral palsy have used the talonavicular coverage angle to assess the amount of forefoot abduction in the planovalgus foot. The authors have not reported on this angle. Did the authors use a standardized measurement technique, as described by Davids et al. [1], to measure their radiographs?

5. In the gait parameters part of the results section, the authors comment that no significant changes were seen in the gait parameters. However, they have only reported on the temporospatial gait parameters. They have not provided any kinetic or kinematic data.

6. Dynamic pedobarography has been reported as being an economical and objective tool to evaluate the outcome of surgeries to correct foot deformities [2,3]. Do the authors use this in their evaluation protocol?

7. How did they make the decision to fuse only the talonavicular joint? What about the naviculocuneiform and the medial cunio-first metatarsal joint?

I would appreciate a response from the authors.

Thanking you,

Yours sincerely,

Dr. Mohan V. Belthur, MD

\section{References}

1. Davids JR, Gibson TW, Pugh LI (2005) Quantitative segmental analysis of weight-bearing radiographs of the foot and ankle for children: normal alignment. J Pediatr Orthop 25:769-776

2. Chang CH, Miller F, Schuyler J (2002) Dynamic pedobarograph in evaluation of varus and valgus foot deformities. J Pediatr Orthop 22:813-818

3. Park KB, Park HW, Lee SK, Joo SY, Kim HW (2008) Changes in dynamic foot pressure after surgical treatment of valgus deformity of the hindfoot in cerebral palsy. J Bone Joint Surg Am 90:1712-1721 